\title{
EXPERIMENTAL BACTERIAL MASTITIS IN THE MOUSE
}

\author{
R. L. Chandler \\ Agricultural Research Council, Institute for Research on Animal Diseases, \\ Compton, Nr Newbury, Berkshire

\section{Plates XXIII AND XXIV}

ProBLEMS of husbandry, including management and costs, have always caused difficulties in the use of cows for the study of bovine mastitis. Goats and sheep have been used as a substitute, but with these species husbandry problems are also considerable. A laboratory animal model for studying mastitis that was inexpensive and readily maintained by laboratories with standard facilities would be of considerable importance. This paper describes the production of experimental mastitis in mice by intramammary inoculation. Organisms that are commonly associated with naturally occurring bovine mastitis were inoculated and the macroscopic and histological responses observed closely resembled those that occur in the cow. The model provides a convenient system for fundamental studies and quantitative experiments on mastitis prior to final assessment in cattle.

\section{MATERIALS AND METHODS}

Mice. The BSVS strain, maintained as a closed colony at this Institute for several years, was used. In preliminary experiments mice that had produced more than one litter and were at varying stages of lactation were taken from the breeding stock. In later experiments mice that had borne only one litter and were at the 10th-15th day of lactation were used to provide a more constant standard. Offspring were removed from the lactating mice and disposed of about 1-2 hr before inoculation.

Bacteria inoculated. The following bacterial species were used in infection experiments: Staphylococcus aureus (strain S57), Streptococcus agalactiae (strains S13 and 122), Corynebacterium pyogenes (strain PM 1), Escherichia coli (strain GS. 1) and Pseudomonas aeruginosa (strain GS. 2). All these strains had originally been isolated from cases of bovine mastitis.

Inocula were prepared from cultures in Todd-Hewitt broth ( $C$. pyogenes in serum broth) and the bacterial suspensions were standardised to Brown's tube no. 8 before further dilution. Bacterial counts were made by the method of Miles and Misra (Miles, Misra and Irwin, 1938). Control inocula consisted of heat-killed organisms, culture filtrate and culture media. The volume inoculated was approximately $0.05 \mathrm{ml}$ per teat.

Intramammary inoculation technique. The mice were lightly anaesthetised with ether and were laid on their backs and to one side on a small cork board so as to present the teats chosen for inoculation (fig. 1). The teats were lightly dabbed with cotton-wool soaked in surgical spirit; this laid down the hair around the teats and facilitated subsequent observation. Hamilton $33 \mathrm{G}$ steel needles (V. A. Howe Ltd, Pembridge Road, London), $\frac{3}{8}$ in. $(1 \mathrm{~cm})$ long with Luer fitting and mounted on $1-\mathrm{ml}$ disposable plastic syringes were employed. During inoculation each teat was viewed through a $\times 10$ bench binocular magnifier (Vickers "Sterimag"). The flaccid teat was held lightly with fine forceps near its tip and the needle positioned at the centre of the tip of the teat; the needle was then

Received 18 June 1969; accepted 7 Aug. 1969.

J. MED. MICROBIOL.-VOL. 3 (1970) 
gently introduced into the teat, and at the same time the teat was drawn upwards on the needle with the forceps. The needle was introduced to about two-thirds of the length of the teat; its correct location along the axis centre could then be observed, and the inoculum delivered while the teat was still held with the forceps. In a high proportion of cases it was observed that the needle penetrated at a point other than the streak canal orifice, and indeed such a situation may have been the rule, but this did not appear to alter the effect of the procedure. Leakage from the teat was not observed after use of the correct procedure.

Each mouse possessed ten teats, six in the thoracic and four in the abdominal group (fig. 2). On each side the three thoracic mammary glands were contiguous and so were the two abdominal mammary glands. The results of inoculation of dyes and observations on the production of mastitis indicated that each mammary gland was anatomically and functionally distinct from its neighbour in the same manner as in the bovine species. The mouse, like the cow, has one teat duct or streak canal per teat. For convenience of recording, the teats were designated as follows: left side, from anterior to posterior, L1, L2, L3 (thoracic group) and L4, L5 (abdominal group); right side, similarly R1, R2, R3 and R4, R5. The abdominal mammary glands were larger than the thoracic ones. Usually, active material was inoculated into teats on the left side (commonly L4 and L3) and control material into the corresponding teats on the right side of other mice of the group.

Clinical observations. Inoculated mice were examined daily for local and generalised reactions. Local reactions ranged from darkening of the skin at the base of the teat to obvious swelling of the affected mammary gland. Generalised reactions, when they occurred, included ruffled coat, hunched appearance, weakness and in some cases death.

Post-mortem examination. Mice were killed with ether and laid on their backs, and the skin was opened along the mid-line and everted to display the mammary glands (fig. 2). Affected mammary glands could be distinguished from the control glands by swelling, discoloration and exudation or abscess formation.

Histological procedures. Histological examinations were made on the majority of inoculated mice. Each mammary gland to be examined was dissected, fixed in neutral buffered formaldehyde and stained with haematoxylin and eosin and, in some cases, by Gram's method also.

Bacteriological procedures. Bacterial counts were made on the mammary glands in appropriate cases by grinding in a Griffith's tube with a measured volume of fluid, followed by dilution in series, culturing and counting.

\section{RESULTS}

\section{Inoculation of Staphylococcus aureus}

Introductory experiment. Staph. aureus, resuspended in original culture medium and standardised to Brown's tube no. 8, was injected into the teats of each of two lactating mice from the breeding stock, at a dose of $0.05 \mathrm{ml}$ per teat. One mouse, into two of whose teats Staph. aureus had been inoculated, showed swelling and hardening of the inoculated mammary glands and a ruffled coat $24 \mathrm{hr}$ after inoculation; when it was killed at $48 \mathrm{hr}$ the experimental mammary glands were enlarged, and histological examination (fig. 3) showed acute mastitis characterised by neutrophil infiltration, early necrosis, and the presence of numerous cocci. The other mouse, three of whose teats had been used for inoculation, showed similar symptoms at $24 \mathrm{hr}$ and died at $48 \mathrm{hr}$ with lesions of acute mastitis in all the experimental mammary glands.

Titration. A titration experiment was then done (table I) on mice that had produced only one litter and were at the 10th-15th day of lactation. Ten-fold dilutions of a stock bacterial suspension standardised to Brown's tube no. 8 


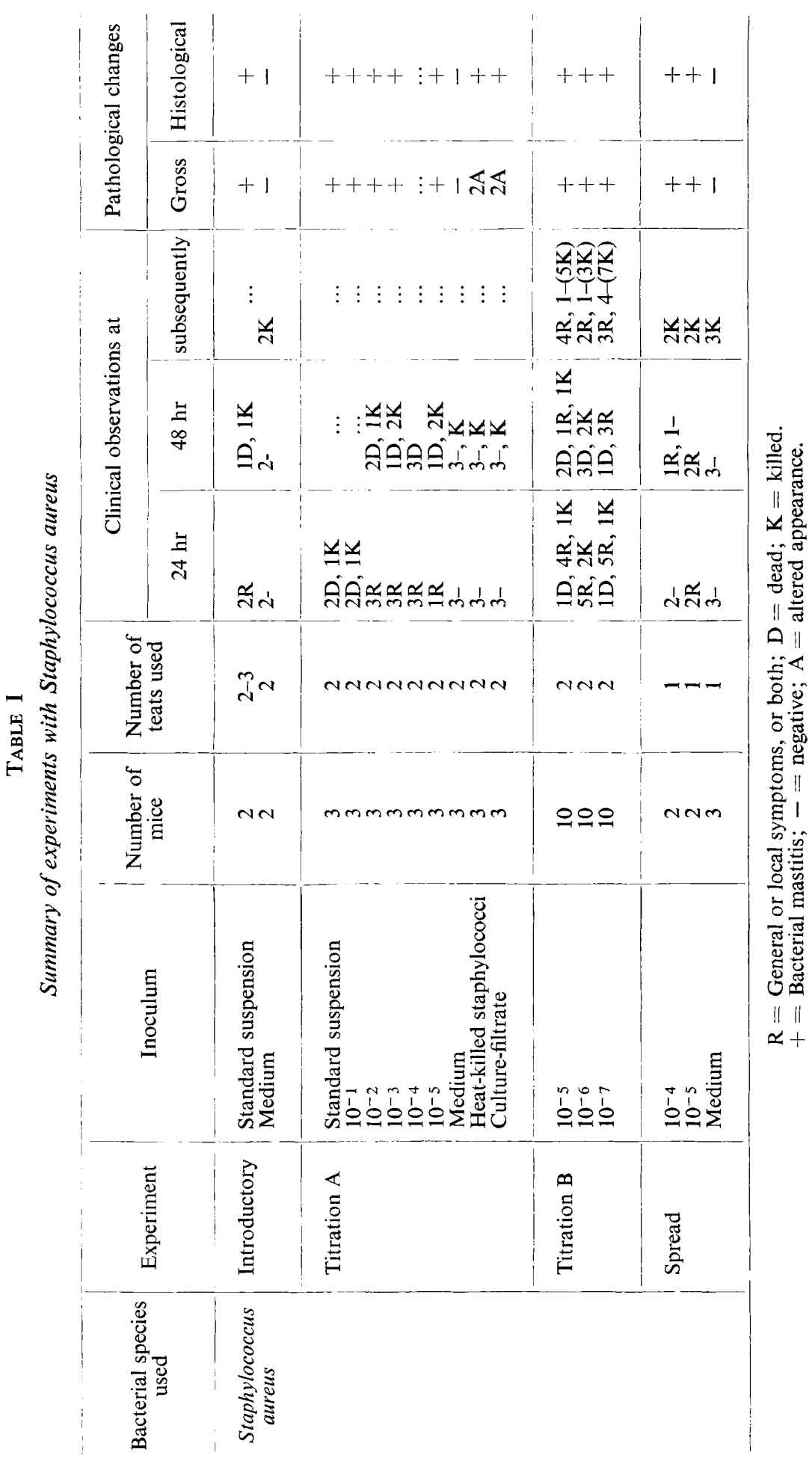


(viable count $5 \cdot 5 \times 10^{8}$ organisms per $\mathrm{ml}$ ) were injected into teats L3 and L4. Heat-killed organisms, culture-filtrate and medium were injected into teats on the control side.

Twenty-four hours after inoculation deaths occurred in the groups of mice given the two highest concentrations of organisms, and mastitis, often accompanied by general illness, occurred in all the other groups of the dilution series, including the $10^{-5}$ dilution group, in which only $2.75 \times 10^{2}$ organisms had been inoculated into each teat.

Forty-eight hours after inoculation, mice in the $10^{-5}$ dilution group were very ill, and post-mortem examination showed acute mastitis with hard, swollen mammary glands, discoloration and extravasation of blood-stained exudate. Histological examination confirmed the acute mastitis and the presence of numbers of cocci. Bacteriological counts assessed the average number of viable cocci per affected mammary gland as $2.4 \times 10^{8}$, whereas the number of cocci inoculated into each mammary gland had been $2.75 \times 10^{2}$. At this time the surviving mice in the higher dose groups were very ill and were killed for examination; acute mastitis confirmed by histological examination was recorded.

Mice in the control groups remained normal in appearance. In the group inoculated with heat-killed staphylococci, post-mortem examination at $48 \mathrm{hr}$ showed that the inoculated mammary glands were more vascular than their controls; histological examination showed an early mastitis resembling that produced with live organisms, but the cocci seen in Gram-stained sections were reduced in number and dispersed. In the control group receiving filtrate, the post-mortem examination showed little evidence of mastitis. Histological examination, however, showed an early mastitis with absence of cocci in one mouse. There was some involution in the second mouse and indications of stasis of the milk-duct contents in the third animal.

It was evident that the BSVS mice were very susceptible to small numbers of this strain of staphylococcus, and a further experiment was made using similar mice in groups of 10 , and ten-fold dilutions of cocci (table I, titration B). The stock suspension of cocci used in this experiment had a viable count of $7 \times 10^{8}$ per $\mathrm{ml}$, i.e., similar to that of the first titration experiment.

Twenty-four hours after inoculation, the mice in each of the dilution groups showed a progressive range of findings varying from normal appearance, swollen mammary glands only, general illness with swollen mammary glands and in two cases death. Some of the sick mice were killed; the post-mortem examination showed mastitis, which was confirmed by histological examination.

Forty-eight hours after inoculation a similar situation prevailed. At this time and during the following 12 days individual sick mice were killed; they all showed active mastitis.

The experiment was terminated 2 wk after inoculation, at which time three mice in the $10^{-7}$ dilution group, one in the $10^{-6}$ dilution group and three in the $10^{-5}$ dilution group appeared normal; post-mortem examinations revealed inactive mammary abscesses in a proportion of the mice, indicating residual lesions resulting from previous infection. 
These results suggested that the BSVS mice were very susceptible to intramammary infection with small numbers of the strain of staphylococcus used; a proportion of cases occurred at the lowest titre equivalent to 3.5 viable units per inoculated teat.

Spread between mammary glands. In the course of the above experiments there was no evidence of spread of infection from one mammary gland to another, within the time of duration of the experiments. In order to study this further, four additional mice of the same category were inoculated, two with a $10^{-4}$ dilution of stock suspension equivalent to $1.2 \times 10^{3}$ viable units per teat dose, and two with a $10^{-5}$ dilution equivalent to $1.2 \times 10^{2}$ viable units per teat dose. In each mouse only one teat, L4, was used.

Twenty-four hours after inoculation two mice showed swollen mammary glands together with general illness and were killed to demonstrate mastitis in mammary gland L4. One mouse was examined bacteriologically by culture of mammary glands L3 and L5; L3 gave negative culture results, L5 a positive result for staphylococci. Another mouse was examined histologically in regard to L3, L5, R4 and R5; some lymphocyte infiltration was observed in L5, but otherwise no mastitis was observed in the neighbouring mammary glands. Three control mice inoculated with broth culture medium did not develop mastitis. It was evident that in experiments of comparatively short duration spread of infection from one mammary gland to its uninoculated neighbour was not likely to be a serious complicating factor.

\section{Inoculation of Streptococcus agalactiae}

General experiments. Mice from the breeding stock were used in these studies (table II). In an introductory experiment strain 122 resuspended in its original broth medium and standardised to Brown tube no. 8 was inoculated into the L4 mammary gland of a mouse. No clinical signs were visible $48 \mathrm{hr}$ after inoculation when the mouse was killed for examination, but histological examination showed an intramammary abscess with large numbers of Grampositive occci.

Strain S13 was similarly inoculated into L3 and L4 mammary glands of two mice. Transient swelling of a mammary gland was recorded in one of the mice. At post-mortem examination, 6 days after inoculation, lesions resembling abscesses were noted in the inoculated mammary glands and were confirmed histologically.

In two further experiments both strains of streptococcus were used, together with controls consisting of the heat-killed strains and broth medium. Four mice, in each of which strain S13 was inoculated into L3 and L4, and three mice into which strain 122 was similarly inoculated developed mastitis. At post-mortem examination the affected mammary glands were enlarged and discoloured, with a grey or mottled red appearance in all cases except one, where there was a large abscess at the base of the teat. Histological examination (fig. 4) showed milk stasis, acini filled with cells and debris, and infiltration with neutrophils and lymphoid cells; numerous cocci were present. Similar mastitis 


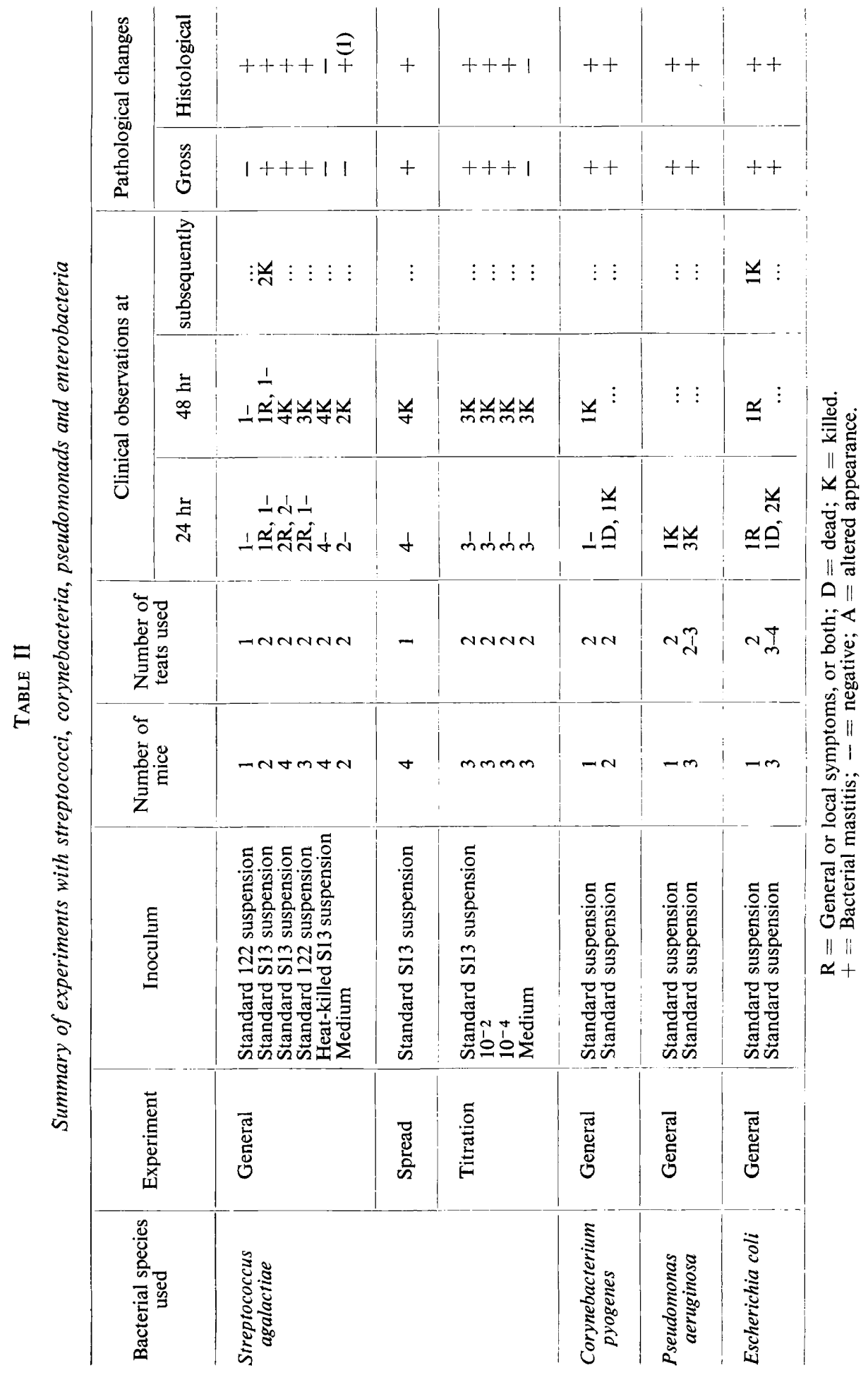


was not seen in four control mice into which heat-killed organisms had been inoculated, but in three of the four cases histological examination revealed a relatively mild reaction characterised by the presence of macrophages in some acini and associated with small numbers of Gram-positive cocci.

Spread between mammary glands. Spread of mastitis from inoculated to uninoculated neighbouring mammary glands was not found in these experiments with streptococci. In a further study in four mice, streptococci were inoculated into L4 only. Forty-eight hours later two of the mice had developed evident mastitis of L4 and were killed; cultural examinations of the neighbouring mammary glands of one of these mice were negative, whilst histological examination of the other mouse showed mastitis in L5 associated with Gram-positive cocci. Five days after inoculation the other two mice were killed and examined and mastitis was noted in the inoculated mammary glands only.

Titration experiment. In a titration experiment (table II) the standard suspension of the S13 strain (equivalent to $1.4 \times 10^{7}$ viable units per teat dose), a $10^{-2}$ dilution $\left(1.4 \times 10^{5}\right.$ viable units per teat dose $)$ and a $10^{-4}$ dilution $\left(1.4 \times 10^{3}\right.$ viable units) were inoculated into groups of three mice; a group into which culture medium was inoculated served as controls (table II). Forty-eight hours after inoculation general and local clinical reactions were seen in the infected groups and all were killed for examination. Mastitis was noted at all three dilutions and was confirmed histologically. Bacterial counts of affected mammary glands gave averages of $6 \times 10^{7}$ viable units in the standard suspension group, $3 \cdot 2 \times 10^{8}$ in the $10^{-2}$ dilution group and $2.5 \times 10^{8}$ in the $10^{-4}$ dilution group. It was clear that bacterial multiplication had occurred after inoculation.

In the course of this experiment it was noted that where L3 mammary glands were inoculated, and mastitis subsequently developed, there was some difficulty in discerning the precise boundary of the lesion in relation to the boundary of the mammary gland. The possibility of local spread to the other thoracic mammary glands on the same side was therefore not determined in these cases.

\section{Inoculation of Corynebacterium pyogenes}

A mouse into which a suspension of $C$. pyogenes resuspended in its original culture medium and standardised to Brown's tube no. 8 was inoculated developed visible swelling of both inoculated mammary glands $48 \mathrm{hr}$ after inoculation and was killed for examination (table II). The mammary glands were enlarged and haemorrhagic and associated with a subcutaneous gelatinous exudate. Histological examination (fig. 5) showed areas of necrosis, infiltration with neutrophils and mononuclear cells, pronounced congestion with some haemorrhage, and large numbers of Gram-positive diphtheroid bacilli.

In a further experiment $C$. pyogenes was injected similarly into three mammary glands (L3, L4 and L5) of each of two mice. Twenty-four hours after inoculation one mouse died; the other mouse showed some swelling of the mammary glands together with general symptoms and was killed. Post-mortem and histological examinations confirmed the presence of mastitis. 


\section{Inoculation of Pseudomonas aeruginosa}

A mouse into which a suspension of Ps. aeruginosa standardised to Brown's tube no. 8 had been inoculated, developed local and general symptoms in $24 \mathrm{hr}$ and was killed for examination. The affected mammary glands were enlarged and haemorrhagic. Histological examination (fig. 6) showed extensive necrosis, some cellular infiltration, marked congestion with haemorrhage and numerous bacteria.

$P s$. aeruginosa was inoculated into two mammary glands of one mouse and into three mammary glands in each of the two others. Twenty-four hours after inoculation the inoculated mammary glands were swollen and tender, and early general symptoms were seen; the mice were killed. Post-mortem and histological examinations confirmed mastitis.

\section{Inoculation of Escherichia coli}

A mouse into whose mammary glands an Esch. coli suspension standardised to Brown's tube no. 8 had been inoculated showed slight swelling of the two inoculated mammary glands $24 \mathrm{hr}$ later (table II). Five days after inoculation a small scar indicating a ruptured abscess was seen at the base of each inoculated mammary gland and the mouse was killed. Post-mortem examination showed an abscess of one mammary gland; the other was enlarged and oedematous and histological examination showed areas of necrosis and oedema.

Esch. coli was inoculated into four mammary glands of one mouse and into three mammary glands in each of another two. Twenty-four hours later the mouse that had four teats inoculated had died; the other two were sick and were killed for examination. The affected mammary glands were enlarged, reddish brown in colour and oedematous. Histological examination (fig. 7) showed varying degrees of change throughout the mammary tissue, ranging from swelling of the acinar cells to necrosis. Neutrophil infiltration was minimal; Gram-negative bacilli were numerous; microscopic abscesses and haemorrhages were also observed. The milk ducts were packed with large masses of cells and interacinar oedema was evident (cf. fig. 8).

\section{Discussion}

The procedure developed for intramammary inoculation is simple and reproducible. It was evident that lactating mice from breeding stocks could be utilised, but it is obviously preferable that more standard conditions should be used, such as mice that have had one litter only and are at a certain lactation state, e.g., 10-15 days post-partum, for optimum development and activity of the mammary glands. The mice of the latter category used in these experiments were in most cases carrying their second litter; an improved standard is planned for future experiments by taking away the males as soon as the females are seen to be carrying their second litter. A second management aspect was that the sucking mice were taken away from the lactating females one or two hours before inoculation of the latter and were not returned, so that the mammary glands were no longer milked. If it were desirable for milking to be continued 


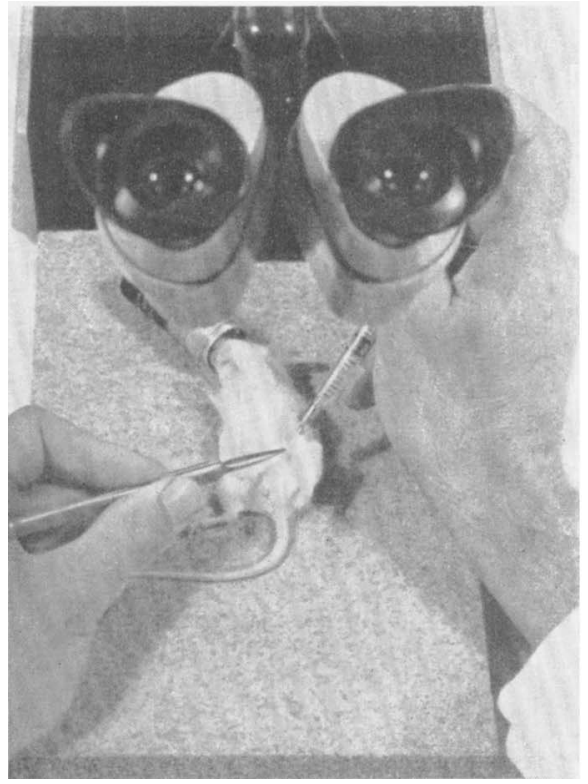

FIG. 1.- Inoculation procedure, The anaesthetised mouse, binocular viewer, $1 \mathrm{ml}$ syringe with $33 \mathrm{G}$ needle and fine forceps are shown.

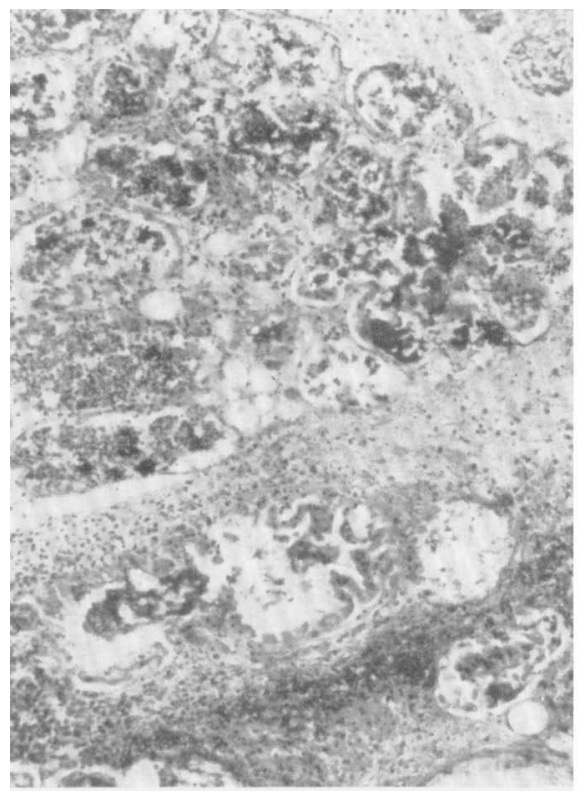

FIG. 3.-Mastitis due to Staphylococcus aureus. Acini and ducts contain neutrophils and cellular debris and there are areas of necrosis and general neutrophil infiltration. Haematoxylin and eosin (HE). $\times 125$.

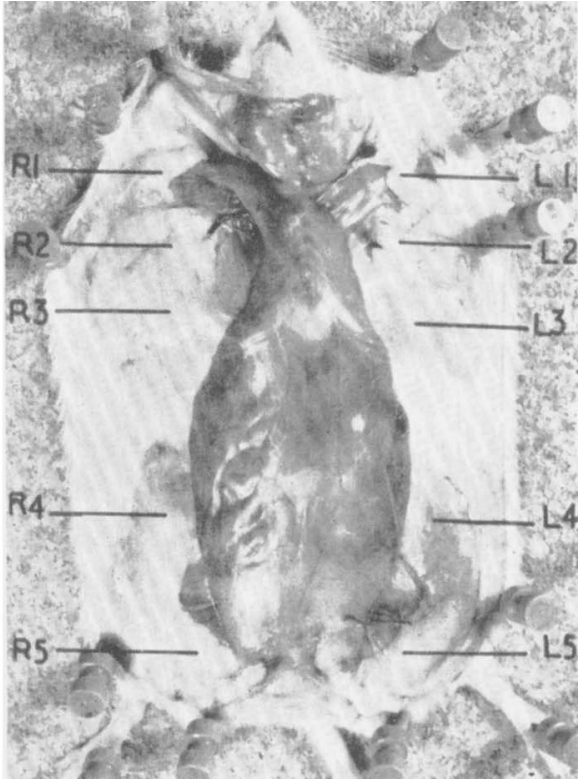

Fig. 2.--Mammary glands displayed by opening and everting the skin along the ventral mid-line. Individual mammary glands are indicated by the code letter and number. Early mastitis due to Psendomonas aeruginosa is seen in mammary gland L4.

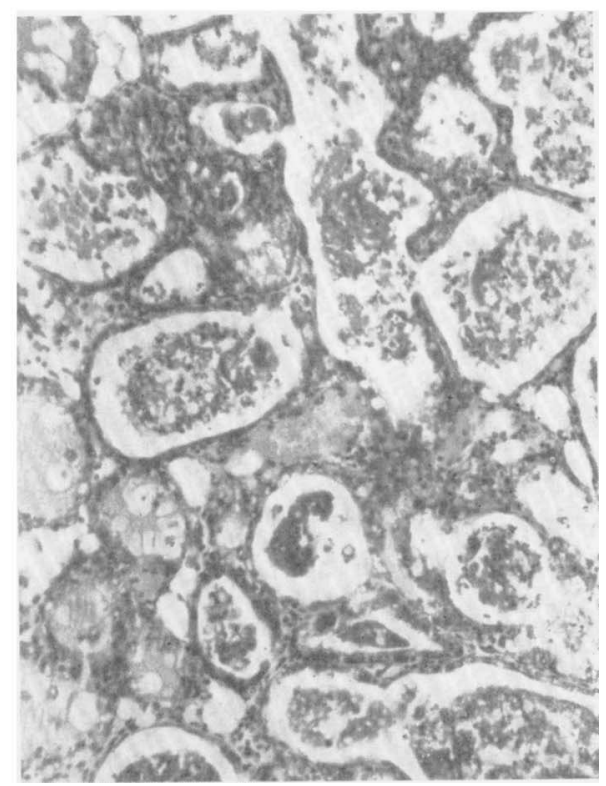

FIcr. 4.--Mastitis due to Streptococcus agalactiae, showing acini filled with cells and debris and tissue infiltration with neutrophils and lymphoid cells. HE. $\times 125$. 


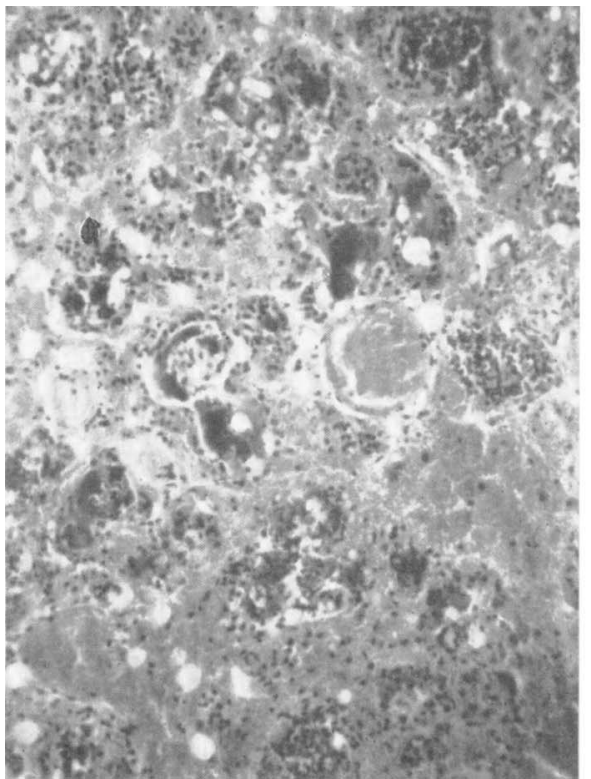

FIG. 5.--Mastitis due to Corynehacterium pyogenes, showing congestion, haemorrhages, necrosis and infiltration with neutrophils and mononuclear cells. HE.

125.

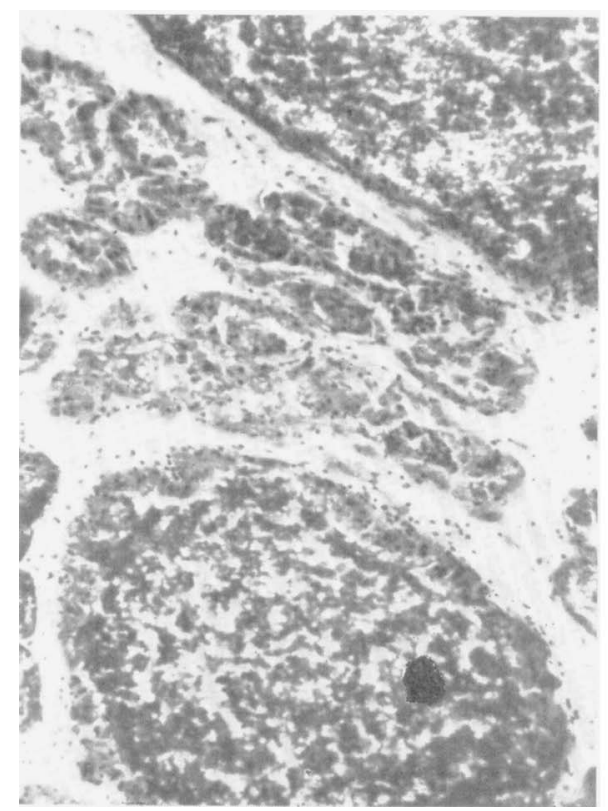

FIG. 7.--Mastitis due to Escherichia coli. The ducts in particular are grossly distended with debris and there is interacinar oedema: neutrophil infiltration is minimal. HE. $\times 125$.

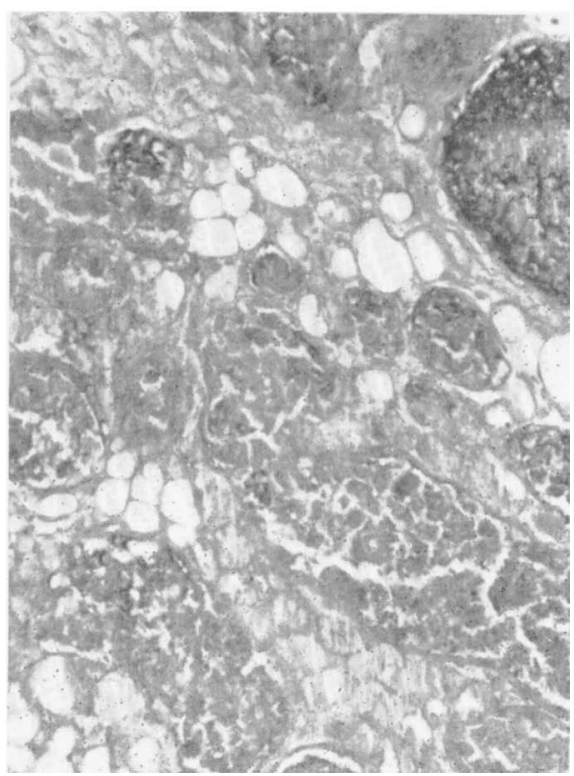

Fig. 6.- Mastitis due to Pseudomonas aeruginosa. Acini and ducts are grossly distended with cellular debris and there is tissue necrosis. HE. 125.

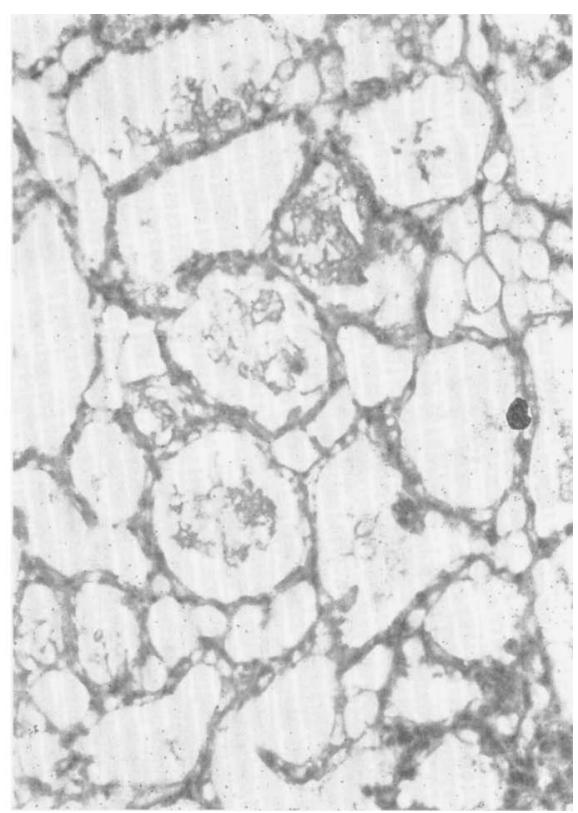

FIG. 8. - Control uninoculated mammary gland showing normal acini and ducts, some containing milk products. HE. 125 . 
this could be attempted by returning the sucking mice or by use of laboratory apparatus designed for the milking of laboratory animals (Temple and Kon, 1937).

The BSVS strain of mice used was evidently very susceptible to infection, particularly with staphylococci, by the intramammary route. This is not unexpected, and it is possible that different strains of mice have different degrees of susceptibility.

The experiments with staphylococci showed that mastitis could be produced readily and by a comparatively small number of organisms. This suggests that the model would be very suitable for, e.g., detection of degrees of conferred artificial immunity and the protective or curative effects of pharmaceuticals, with the proviso that final assessment would be carried out on cattle. The course of the infection, the results of cultural examinations and the use of controls made it clear that the mastitis was associated with progressive multiplication of staphylococci and was not merely the response to the original introduction of the cocci. Since staphylococcal mastitis is one of the most important forms of bovine mastitis a model for studies in this respect is likely to be of considerable interest.

The experiments with streptococci indicated that the degree of susceptibility to infection with this organism was more variable and lower than that experienced with staphylococci. This has its parallel in the bovine species where experimental infection with streptococci by the intramammary route also produces variable results (Howell et al., 1954). In this respect, Pattison (1949) has reported the general pathogenicity for mice of streptococci of bovine origin injected intraperitoneally or intravenously.

The experiments with C. pyogenes, Ps. aeruginosa and Esch. coli indicated that mastitis could be produced in mice by these species by intramammary inoculation. These organisms are also the cause of bovine mastitis and, as in the cases of the other bacterial species studied here, had originally been isolated from cases of that disease. The character of the histological changes produced by the different bacterial species in the mouse resembled those produced by the same species in cattle (Jubb and Kennedy, 1963).

I usually inoculated organisms into two mammary glands per mouse. This had the advantage that any spread to immediate neighbours in the thoracic or abdominal group, or spread between groups of mammary glands on one side or both sides of the mouse, could be recorded. There was little evidence that such spread would be a complicating factor in experiments of short duration. The size of the mouse mammary gland proved to be very convenient for histological and other studies requiring examination of the whole gland. It will be appreciated that, as in the case of results obtained using laboratory models for other diseases, these findings should finally be assessed and confirmed in the natural host.

\section{SUMMARY}

A simple technique was developed for the intramammary inoculation of mice. Experimental mastitis was produced in lactating mice with strains of 
Staphylococcus aureus, Streptococcus agalactiae, Corynebacterium pyogenes, Pseudomonas aeruginosa and Escherichia coli that had originally been isolated from cases of bovine mastitis. There was evidence that the spectrum of pathogenicity of the different bacterial strains in the bovine species was to some extent paralleled by differences in pathogenicity in the mouse. Mastitis could be produced in BSVS mice by the inoculation of comparatively small numbers of staphylococci. The appearance and histology of the affected mammary glands resembled mastitis produced in the bovine species by the bacterial species concerned. The size of the mouse mammary gland was very convenient for histological and other studies requiring examination of the whole gland. Experimental mastitis in the mouse provides a useful model for certain fundamental studies and for experiments, such as those of a screening nature, prior to studies on cattle.

I wish to express my thanks to Messrs P. Dennis and A. Soames for technical assistance, and to $\mathrm{Mr}$ I. Jebbett for photographic assistance.

\section{REFERENCES}

Howell, D. G., Pattison, I. H., Holman, $\quad$ 1954. J. Comp. Path. Ther., 64, 335.

H. H., AND SMITH, I. M.

Jubb, K. V. F., AND Kennedy, P. C. 1963. Pathology of domestic animals, New

Miles, A. A., Misra, S. S., AND Irwin, 1938. J. Hyg., Camb., 38, 237.

J. O.

PATTISON, I. H. . . . . . . . . . . 1949. Ibid., 47, 159.

Temple, P. L., AND Kon, S. K. . . 1937. Biochem. J., 31, 2197. 